1." M W Johnson, 'K Lithgo, 'T Price. 'Gastroenterology, Luton \& Dunstable University Hospital, Luton, UK

Introduction The incidence and prevalence of inflammatory bowel disease in Asia, is generally lower than what we see in the West, however, in recent years there has been a significant increase. Currently there is little information available regarding the impact of ethnicity on inflammatory bowel disease (IBD) prevalence within the UK. One study has suggested that young Asians born in Britain are at a significantly higher risk of developing IBD than the indigenous European population.

Objectives To assess the prevalence and disease distribution amongst our ethnically diverse local population.

Methods Data from the Office for National Statistics 2010 was used to establish the general make up of the local population. The regional IBD excel database was analysed for differences in the ethnic diversity seen in our IBD cohort as compared to that predicted within the local population. In addition, differences in disease type were also assessed for between the different IBD ethnic groups, using the Mann Whitney unpaired t-test.

Results The local population is made up by White 68\% Asian 19\% (Indians 4.4\% Pakistanis 10.6\% and Bangladeshis 4\%), Black 7\%, Mixed 3\% and Chinese + others 3\%. The IBD database contains 2755 patients, but 270 did not want to disclose their ethnicity. Of the remaining 2485, 2059 (83\%) were White, 303 (12\%) were Asian, (Indians 111, Pakistanis 133 and Bangladeshis 35), 92 (4.5\%) were Black, 28 (1\%) were Mixed and 3 were Chinese. IBD was less common within the Asian cohort, but there was a statistical differences seen in the type of IBD suffered by the local Caucasian and Asian populations $(p=0.0141)$. Asian patients had proportionally more UC and less Crohn's, with the exception of the Bangladeshis who had a higher (but not statistically significant) prevalence of Crohn's disease.

Conclusion IBD is less common in the Asian community. Proportionally UC is more frequently experienced than Crohn's within the Asian population. This may be related to the known increased genetic predisposition of Southern Asians (Indians, Pakistanis and Bangladeshis) to ulcerative colitis.

Disclosure of Interest None Declared.

\section{PWE-098 MECHANISMS UNDERLYING THE DEVELOPMENT OF CHRONIC INFLAMMATION IN INFLAMMATORY BOWEL DISEASE: DEFINING THE ROLE OF THE RAGE PATHWAY USING COMPUTATIONAL AND BIOLOGICAL ANALYSIS STRATEGIES}

doi:10.1136/gutjnl-2013-304907.386

1." M Bramhall, ${ }^{2} \mathrm{~N}$ Han, ${ }^{3 R}$ Haggart, ${ }^{3} \mathrm{~J}$ Wilson, ${ }^{1} \mathrm{~A}$ Brass, ${ }^{2} \mathrm{~S}$ Cruickshank. 'Computer Science; ${ }^{2}$ Faculty of Life Sciences, The University of Manchester; ${ }^{3}$ Epistem Ltd., Manchester, UK

Introduction Inflammatory bowel disease (IBD) is a chronic inflammatory disease with an estimated annual cost to the NHS of $£ 720$ million. Patients typically present with established disease and this makes it difficult to determine the underlying aetiology: knowledge that would aid early diagnosis and treatment.

Methods To better define factors underlying the development of IBD that might be used as diagnostic aids for treatment/prevention of IBD we have analysed the early immune response in mice that will develop colitis using a validated infection model of colitis. Microarray analyses of colon tissue were conducted using the Puma and Tigre packages for Bioconductor to determine gene expression and investigate the transcription factor pathways involved.
Results Microarray analysis identified an early and rapid increase in expression of the receptor for advanced glycation end-products (RAGE) in colitic prone (susceptible) mice. In contrast, mice that clear the infection (resistant mice) had no increase in RAGE. In addition, the transcription factor analysis revealed a downregulation of colitic protective factors in the RAGE signalling pathway. Immunohistochemistry data showed high RAGE expression in the gut epithelium prior to the onset of colitis. Previous work from our group has shown that epithelial cells promote dendritic cell recruitment associated with resistance and clearance of parasite infection. In the colitis model, infection also induced the rapid recruitment of macrophages and dendritic cells (DC) into the gut and lymph nodes in resistant mice but not susceptible mice. By day 31 post-infection, resistant mice had resolved the infection and inflammation whereas susceptible mice had significantly higher immune cell accumulation and colitis. Current work is addressing the expression of RAGE blocking ligands and the regulation of RAGE in the guts of resistant and susceptible mice.

Conclusion RAGE has been associated with chronic IBD in patients however our data implicates RAGE in the development and propagation of IBD. We propose that the RAGE pathway is an early indicator of IBD and may be useful therapeutically and in determining efficacy of IBD therapy.

Disclosure of Interest M. Bramhall Grant/Research Support from: Epistem Ltd., N. Han: None Declared, R. Haggart: None Declared, J. Wilson: None Declared, A. Brass: None Declared, S. Cruickshank: None Declared.

\section{PWE-099 FAECAL CALPROTECTIN: HELP OR HINDERANCE IN EVALUATING PATIENTS WITH LOWER GI SYMPTOMS}

doi:10.1136/gutjnl-2013-304907.387

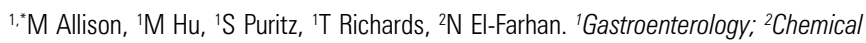
Pathololgy, Royal Gwent Hospital, Newport, UK

Introduction Several studies demonstrate the potential of faecal calprotectin measurement in distinguishing inflammatory bowel disease (IBD) from irritable bowel syndrome (IBS). It is unclear, however, to what extent such measurements alter patient investigation and management over standard history taking, examination and routine blood tests.

Methods We reviewed all faecal calprotectin results from samples submitted by adults not previously known to have IBD between February 2010 and April 2012. Using the health board's Clinical Workstation relevant outpatient letters, results of subsequent investigations and clinical outcomes were reviewed. A calprotectin value of $>60 \mathrm{ug} / \mathrm{g}$ was considered elevated.

Results Cinical data was missing for 13 of the 266 patients. Of 155 with a normal result management was unaltered in 126, of whom 50 were referred for lower GI endoscopy before their result was known. In another 5 patients IBD was later found despite a normal result. A normal result may have obviated the need for colonoscopy or capsule endoscopy in 17 , and otherwise altered management in 12 other patients. Outcomes for the 98 with an elevated result are summarised in the table. Patient management was unaltered in 60 . There were 33 for whom an elevated result prompted colonoscopy and/or capsule endoscopy, and results were normal in 27 . Five others still await colonoscopy or capsule.

Conclusion This study casts doubt on the value of faecal calprotectin in the routine evaluation of patients presenting with lower GI symptoms. Invasive investigations prompted by elevated results proved normal much more often than abnormal. The assay seems 
most helpful in evaluating patients with symptoms suggestive of IBS with abnormal blood tests, and in those in whom previous investigations were equivocal for IBD

Disclosure of Interest None Declared.

\section{Abstract PWE-099 Table}

\begin{tabular}{ll}
\hline OUTCOMES FOR PATIENTS WITH ELEVATED FAECAL & $\begin{array}{l}\mathbf{n}=\mathbf{9 8} \text { of whom } \\
\mathbf{5} \text { await tests }\end{array}$ \\
\hline CALPROTECTIN & \\
\hline Management unaltered & 19 \\
Not pursued ( $\mathrm{n}=14$ ), or did not attend or refused investigation $(\mathrm{n}=5)$ & 25 \\
Further investigation indicated anyway (symptoms/bloods) & 16 \\
Self-limited or other illness & \\
Misleading positive result & 27 \\
Colonoscopy +/- capsule NAD (or insignificant incidental finding) & \\
Helpful positive result & 2 \\
Previous investigations equivocal: IBD later confirmed & 4 \\
Prompted positive capsule, colonoscopy or MR enterography & \\
\hline
\end{tabular}

\section{PWE-100 HOW COMMONLY DOES FAECAL CALPROTECTIN ALTER MANAGEMENT IN PATIENTS WITH I.B.D?}

doi:10.1136/gutjnl-2013-304907.388

1." M Allison, 'M Hu, 'S Puritz, 'T Richards, ${ }^{2} \mathrm{~N}$ El-Farhan. ' Gastroenterology; ${ }^{2}$ Chemical Pathology, Royal Gwent Hospital, Newport, UK

Introduction Faecal calprotectin is often measured in patients with inflammatory bowel disease (IBD). Some believe it is a useful surrogate marker for mucosal healing, and normal values may strengthen the case for stopping biological agents. It is unclear, however, to what extent such measurements alter patient investigation and management over standard history taking, examination and routine blood tests.

Methods We reviewed all faecal calprotectin results from samples submitted by 98 adults with IBD between February 2010 and April 2012. Using the Health Board's Clinical Workstation relevant outpatient letters, results of subsequent investigations and changes of treatment were reviewed. A calprotectin value of $>60 \mathrm{ug} / \mathrm{g}$ was considered elevated.

Results Seventy of the patients (71\%) had an elevated result. Their outcomes are summarised in the table. Most changes in patient management and investigation requests were made at the same visit as the calprotectin request. Elevated results prompted escalation of treatment in 6 patients. Among 28 patients with a normal result, symptoms prompted escalation of treatment ( 6 patients) and colonoscopy ( 3 patients) before knowledge of the result, No endoscopic or radiological investigations or changes of treatment occurred in 12 patients. In no cases was maintence treatment reduced on the basis of a normal result. Measurements seemed to help managment in 7 patients: there were 2 with previous equivocal investigations who may have avoided further tests, and there were 5 whose symptoms were in excess of objective findings who could be reassured about absence of active inflammation).

Conclusion This study casts doubt on the value of faecal calprotectin measurement in the follow-up of most patients with IBD. Normal results assisted in the reassurance of some patients whose symptoms seemed out of proportion to objective evidence of disease activity. There were no instances of a normal result leading to scaling back of maintenance treatment, and none stopped biological agents.

Disclosure of Interest None Declared.

\section{Abstract PWE-100 Table}

OUTCOMES FOR I.B.D. PATIENTS WITH ELEVATED FAECAL

CALPROTECTIN

$\mathrm{n}=\mathbf{7 0}$

Management unaltered

No change in treatment

Further investigation indicated on basis of symptoms and/or blood results $\quad 11$

Treatment escalated on basis of symptoms and/or blood results (one had surgery)

\section{Management altered}

Led to escalation of treatment

Colonoscopy requested (but bloods or WC scan also pointed to disease activity) 2

Prompted MR enterography, which was positive, but treatment left unaltered

\section{PWE-101 PATIENT'S AWARENESS OF THE NEED FOR VACCINATIONS WHILST ON IMMUUNOSUPPRESSIVE THERAPY}

doi:10.1136/gutjinl-2013-304907.389

1."M Widlak, 'R Matharu, 'A Elzubeir, 'J Slater, 'L Wood, 'S De Silva. 'Gastroenterology, Russells Hall Hospital, The Dudley Group NHS Foundation Trust, Dudley, UK

Introduction With the ever increasing use of immunosuppressive therapy for the management of inflammatory bowel disease (IBD) patients are being exposed to infections which can be prevented by vaccinations administered prior to or during therapy. The European Crohn's and Colitis Organisation guidelines currently recommend that IBD patients who receive immunosuppressive medications should be vaccinated yearly with the influenza vaccine and a pneumococcal vaccination 3-5 yearly.

Methods An audit was carried out within our department to assess our patient's knowledge and uptake of such vaccines. Patients with Inflammatory Bowel Disease were identified from an established data base and those receiving immunosuppressive therapy were invited to complete a questionnaire. Data gathered included age, gender, current treatment and awareness of the need for vaccinations. We also gathered data on the number of patients already vaccinated.

Results A cohort of 88 patients on immmunosuppressive therapy was analysed. $61 \%$ of patients were female and $39 \%$ male. Patients ranged between $16-21$ years (11\%), 22-30 years (18\%), 31-50 years (40\%), 51-70 years (24\%) and above $70(7 \%) .59$ (67\%) patients had Crohn's disease, 26 (29.5\%) ulcerative colitis and 3 of them $(3.5 \%)$ had indeterminate colitis. The majority of patients received immunomodulators including Azathioprine or Mycophenolate ( $\mathrm{n}=48$, $54 \%$ ). $13(15 \%)$ were treated with biologics alone (Infliximab or Adalimumab), 21 (24\%) with combination of biologics and immunomodulators and 6 patients $(7 \%)$ received immunomodulators with a reducing dose of steroids.

$77 \%$ of patients were aware of recommended vaccinations but as many as $23 \%$ were not. $42 \%$ were aware of the importance of receiving dual vaccinations, $35 \%$ were only aware of the need for either the influenza or pneumococcal vaccine, with $23 \%$ unaware of the need for either. In this cohort 54 (61\%) patients had already had or were planning to have the influenza vaccine this year. Patients between the ages of 31-50 years had the highest awareness of the recommended vaccines (86\%), with the majority of uptake of vaccines seen in the $31-50$ year group (63\%). Unfortunately $39 \%$ of patients were not receiving recommended vaccinations with more than half (56\%) of patients being unaware of the need to avoid live vaccinations.

Conclusion Our data suggest that a significant proportion of patients within our cohort are still not receiving vaccinations 